\title{
Promoción de la salud ambiental con niñas y niños en escuelas de Córdoba: el mapeo colectivo como herramienta de participación
}

\author{
Mariana Butinof \\ mariana@butinof.com.ar \\ (iD) orcid.org/0000-0002-7228-5186 \\ Universidad Nacional de Córdoba, \\ Argentina
}

Ambiente y extensión universitaria /

Intervenciones

\author{
marianaeandi@fcm.unc.edu.ar \\ (iD) orcid.org/0000-0002-5952-7726 \\ Juan Manuel Laino \\ juanmanuellaino@gmail.com \\ (iD) orcid.org/0000-0002-2481-985X
}

Iohanna Filipi

ifilippi@unc.edu.ar

(iD) orcid.org/0000-0002-8919-1311

RECEPCIÓN: 30/06/21

ACEPTACIÓN FINAL: 20/08/21

\section{Resumen}

Este trabajo, elaborado por un equipo extensionista de docentes y estudiantes de la Universidad Nacional de Córdoba, surge de la preocupación de un grupo de mujeres organizadas en torno a la producción hortícola y la exposición a plaguicidas en el cinturón verde de la ciudad de Córdoba. Así, se diseñó y llevó a cabo un proyecto junto a la escuela primaria local y su comunidad educativa. Este artículo se propone: a) presentar una experiencia de abordaje de la salud ambiental integral con niñas y niños en un entorno escolar; b) reflexionar acerca la utilidad del Mapeo Colectivo como herramienta para la visibilización e intercambio de saberes, experiencias y situaciones-problema de vida cotidianas. Se postula que el proceso llevado a cabo es de utilidad para la gestión colectiva de la salud ambiental integral junto a niñas, niños, sus familias y docentes.

Palabras clave: salud ambiental; mapeo colectivo; escolares; participación; extensión universitaria.
Promoting environmental health with children in schools in Cordoba: collective mapping as a participatory tool

\section{Abstract}

This work, prepared by an extension team of teachers and students from the National University of Córdoba, arises from the concern of a group of women organized around horticultural production and exposure to pesticides in the green belt of the city of Córdoba. The project was designed and carried out together with the local primary school and its educational community. This article proposes: a) To present an experience of approaching integral environmental health with girls and boys in a school environment; $b$ ) Reflect on the usefulness of Collective Mapping as a tool for making visible and exchanging knowledge, experiences and problemsituations in everyday life. It is postulated that the process carried out is useful for the collective management of comprehensive environmental health, together with girls, boys, their families and teachers.

Keywords: environmental health; collective mapping; schoolchildren; participation; university extension.
Promoção da saúde ambiental com crianças nas escolas de Córdoba: mapeamento coletivo como ferramenta participativa

\section{Resumo}

Este trabalho, elaborado por uma equipe de extensão de professores e alunos da Universidade Nacional de Córdoba, surge da preocupação de um grupo de mulheres organizadas em torno da produção hortícola e da exposição a agrotóxicos no cinturão verde da cidade de Córdoba. É assim que um projeto foi desenhado e executado em conjunto com a escola de ensino fundamental local e sua comunidade educacional. Este artigo se propõe: a) apresentar uma experiência de abordagem da saúde ambiental integral com meninas e meninos em um ambiente escolar; b) refletir sobre a utilidade do Mapeamento Coletivo como ferramenta de visibilidade e troca de conhecimentos, experiências e situaçõesproblema do cotidiano. Postula-se que o processo realizado é útil para a gestão coletiva da saúde ambiental integral, junto a meninas, meninos, seus familiares e professores.

Palavras-chave: saúde ambiental; mapeamento coletivo; escolares; participação; extensão universitária.

Para citación de este artículo: Eandi, M. A.; Laino, J. M.; Filipi, J. y Butinof, M. (2021). Promoción de la salud ambiental con niñas y niños en escuelas de Córdoba: el mapeo colectivo como herramienta de participación. +E: Revista de Extensión Universitaria, 11(15), e0016. doi: 10.14409/ extension.2021.15.Jul-Dic.e0016 


\section{Introducción}

La experiencia que se reporta surge de la demanda recibida por nuestro equipo de docentes investigadores extensionistas por parte de un grupo de mujeres organizadas de la comunidad de barrio Guiñazú de la ciudad de Córdoba, Argentina. La solicitud era respecto de la percepción de exposición a plaguicidas utilizados en la producción agrícola (intensiva y extensiva) en ese barrio periurbano y del posible daño a la salud asociado a ello. Así, luego de algunos intercambios, se propuso desde este colectivo la articulación con la comunidad de la Escuela Primaria UNESCO para trabajar participativamente la temática de la salud ambiental integral durante el segundo semestre de 2019.

El proyecto se desarrolló en el área periurbana de la ciudad, territorio heterogéneo producto de la actividad humana, donde coexisten y entran en conflicto lo urbano, lo rural y lo natural (Burel y Baudry, 2002; Díaz Terreno, 2011). Es aquí donde se sitúa el Cinturón Verde de la Ciudad de Córdoba (CVCC), reconocida zona productora de alimentos que provee de hortalizas frescas a la ciudad y la región (Giobellina, 2018). El paisaje ${ }^{1}$ se muestra en un proceso de transición y retracción. Se puede constatar la mixtura entre quintas hortícolas y urbanización: viviendas, comercios, escuelas, industrias y espacios recreativos rodean las huertas (Eandi et al., 2021). En el CVCC se reconocen como amenazas para el sostenimiento de esta actividad productiva, el avance de la urbanización y el de la frontera agraria extensiva (Barchuk et al., 2017). Por otro lado, emergen problemas por la cercanía de las zonas urbanas al territorio agrícola; entre otros problemas ambientales, se han identificado riesgos para la salud de las personas que viven y trabajan en las quintas (Butinof et al., 2014).

En Argentina, el modelo predominante en el sistema productivo hortícola está caracterizado por explotaciones primarias intensivas en cuanto a los factores de producción de la tierra, trabajo, capital y tecnología (Giarracca y Teubal, 2010). Si se compara con el resto del sector agropecuario, demanda veinte veces más insumos por unidad de producción, entre ellos, plaguicidas, lo que implica un riesgo para el ambiente (Babbit, 2010) con impactos negativos en la calidad e inocuidad de los alimentos y la salud de las poblaciones humanas. Durante las dos últimas décadas, desde diversas instituciones y organizaciones se han expuesto problemas derivados de la aplicación de plaguicidas en zonas de vecindad de viviendas, incluyendo la judicialización de su uso. La problemática pareciera estar invisibilizada en el sector salud, cuyos trabajadores presentarían resistencia a abordarlo (Machado et al., 2016), impidiendo así enfocar el proceso salud- enfermedad-cuidados desde este ámbito.

Nuestro grupo de investigación, Grupo de Epidemiología Ambiental del Cáncer y otras Enfermedades Crónicas, Universidad Nacional de Córdoba (UNC) integrado por un equipo interdisciplinario de profesionales —nutrición, medicina, enfermería, bioquímica, biología, psicología, estadística, ciencias ambientales, entre otras- realizó sucesivas aproximaciones al territorio del CVCC a partir de metodologías participativas con distintos actores en el contexto de vida y trabajo en este cinturón verde. Fue posible reconstruir el escenario de

\footnotetext{
1) Para el arquitecto Javier Maderuelo, en su libro El Paisaje, génesis de un concepto, en la actualidad se presentan dos interpretaciones extremas, una desde una "visión positivista de las ciencias naturales" y otra "la interpretación subjetiva desde la creación artística", y en una posición intermedia, desde las ciencias humanas, que aceptan trabajar el paisaje incluyendo "algunos grados de subjetivismo como variables de trabajo", en lo particular se refiere a cierta rama de la geografía, aludiendo a la geografía cultural. Matus (1987) denomina "paisaje" a aquello que está fuera de la situación del actor o sujeto, y al que solo se puede contemplar sin intervenir.
} 
exposición a plaguicidas en el marco de los procesos productivos y reproductivos cotidianos de las Familias Hortícolas (FH) y reconocer al contexto hortícola del CVCC como multidimensional y complejo. De esta manera, se pusieron en marcha diferentes estrategias de encuentro orientadas a promover transformaciones y reducir riesgos con algunos colectivos (Eandi et al., 2018).

Los Procesos Salud Enfermedad Cuidados (PSEC) se constituyen como producto de la organización de la sociedad, de la estructura de los grupos sociales, de su relación con el entorno y el ambiente y, por consiguiente, de la inserción de los sujetos en la sociedad y de la accesibilidad a la salud (Bertolozzi y De la Torre Ugarte Guanillo, 2012; Gómez López y Rabanaque Hernández, 2000). En términos de Pedro Luis Castellanos (1990), la salud de una población es determinada por un proceso intrincado y socialmente definido en el que existe un movimiento dialéctico entre dimensiones simples y complejas, desde la expresión de los modos de vida de una sociedad (fuerzas productivas, organización económica y política, formas de relacionarse con el ambiente), pasando por las condiciones de vida de la comunidad comprendidas por los procesos de reproducción social, y hasta las formas de vida y conductas individuales, estilos de vida familiares y modos de existencia de las personas. Siguiendo esta línea de pensamiento, Jaime Breilh (2013) postula que la salud de las comunidades es la resultante del movimiento dialéctico entre procesos protectores y situaciones problema en un territorio/tiempos definidos; y el cuidado de la salud, una construcción histórica y social en la cual los vínculos entre las poblaciones y el ambiente intervienen decisivamente, tornándose determinantes del proceso de salud-enfermedad. Desde la perspectiva de los determinantes sociales de la salud, David Briggs (1999) expone que estos comprenden múltiples aspectos, entre ellos, los sanitarios, comportamentales y naturales, e incluyen la violencia, la pobreza, el hacinamiento, la desnutrición, las buenas o malas prácticas de manejo de los recursos naturales y hasta el correcto etiquetado de productos alimenticios, entre tantos otros. Desde estas miradas, el territorio deja de ser meramente el lugar geográfico de ocurrencia de hechos en salud para ser el escenario donde se expresan los movimientos dialécticos entre distintas dimensiones. $Y$ en orden a lo mencionado anteriormente y con el fin de prevenir, investigar y atender aquellos PSEC vinculados con factores ambientales y otros que incrementan la vulnerabilidad de las poblaciones, el abordaje desde la salud ambiental permite incluir múltiples dimensiones para el estudio de las exposiciones ambientales y la salud de las poblaciones (Organización Panamericana de la Salud —OPS—, 2005).

El concepto de salud ambiental evolucionó con el tiempo. La Organización Mundial de la Salud (OMS, 1972) lo vinculó con el control de los procesos químicos, físicos y biológicos, influencias o factores que ejercían efecto directo o indirecto significativo en la persona y la sociedad. En el año 1989 este organismo revisó el concepto y distinguió una parte a la que denominó sustantiva (aspectos de salud determinados por el medio ambiente), y otra parte, activa, en la que contempló los conceptos de evaluación y control (Ordoñez, 2000). Nicholson (2001) planteó que el concepto moderno de salud ambiental es polisémico y amplio y que se ha transformado a partir de los paradigmas ecocéntricos, por los cuales el ambiente es considerado un todo viviente e interconectado, u homocéntricos, que consideran que lo bueno para el individuo es bueno para la sociedad, y lo supremo, que es aquello bueno para un mayor número de personas (ética utilitarista) (Kleffel, 1996). En síntesis, podemos decir que la salud ambiental se ocupa de estudiar las arduas redes causales involucradas 
en la relación entre seres humanos y ambiente y busca entender cómo el ambiente afecta las condiciones de salud sin dejar de lado la forma en que los humanos afectan el ambiente (Plowright et al., 2008).

En cuanto a las problemáticas de la salud ambiental, el informe pionero del National Research Council (NRC, 1993) "Pesticides in the Diets of Infants and Children", señaló la necesidad de reconocer a las niñas y los niños como una población que requiere atención diferencial: tienen una excepcional vulnerabilidad a los efectos agudos y crónicos de los peligros ambientales y son desproporcionadamente susceptibles en comparación con las/os adultas/ os. La exposición a plaguicidas fue identificada como uno de los principales peligros para la salud infantil (Wild y Kleinjans, 2003; Landrigan et al., 2004). Ello, debido a sus características fisiológicas, condición de sujetas/os en continua formación y desarrollo, diferente metabolismo (menor capacidad para descomponer, excretar, activar o desactivar los plaguicidas), conducta normal exploratoria (suelen llevarse a la boca las manos y objetos que pueden estar contaminados y juegan en el suelo), dependencia de adultas/os, y vivir en condiciones de pobreza en mayor proporción que estas/os (Galvez et al., 2010).

El proyecto que se expone en este trabajo se enmarca en una concepción de la extensión universitaria que propicia el encuentro entre diferentes actores e intereses para la producción de conocimientos, articulando de manera crítica los aportes de la ciencia y del saber popular, con el fin de reorientarlos hacia la acción transformadora de la realidad. Entendiendo que extensión implica necesariamente una acción que se desarrolla en el territorio y que la construcción de saberes, el diálogo de los mismos, se produce en lo social (Bentolila, 2018).

A través de metodologías participativas promueve intercambios constructivos entre equipos de investigación y comunidades en los que se abordan conjuntamente las etapas del proceso investigativo y de intervención social; a partir de un diálogo que concede un rol activo a la comunidad, y estimula su participación en el diagnóstico y resolución de sus necesidades. Durante el mismo, se promovió la construcción participativa de información ambiental, por parte de niñas y niños en su entorno educativo, posicionándolos en tanto ciudadanos activos de ciencia la colectiva, cuyos conocimientos pueden ser utilizados para promover la salud ambiental en su contexto (Boulos et al., 2011; Castell, 2015; Lupton, 2015). Se considera a la ciencia ciudadana un instrumento para la educación informal y formal (Senabre et al., 2018).

El propósito de este artículo es presentar una experiencia de abordaje de la salud ambiental integral con niñas y niños en un entorno escolar y reflexionar acerca la utilidad del mapeo colectivo como herramienta para la visibilización e intercambio de saberes, experiencias y situaciones-problema de la vida cotidiana, jerarquizando las expresiones de las niñas y los niños.

El artículo se estructuró de acuerdo con el proceso realizado, es decir, describe inicialmente la metodología de trabajo, luego los conceptos construidos y lo visibilizado de modo individual y colectivo, y finaliza con las reflexiones y conclusiones.

\section{Construyendo la mirada colectiva con niñas y niños en la escuela}

El proyecto se desarrolló durante el año 2019 en la Escuela Primaria UNESCO de barrio Guiñazú, asentado en el CVCC. Fueron parte del mismo 100 niñas/os y sus respectivos equipos docentes, como también las familias pertenecientes a esta comunidad educativa, 
quienes se involucraron en el proceso, acompañaron las producciones desde el hogar y participaron del último taller. El equipo extensionista estuvo integrado en esta oportunidad por 15 docentes investigadores y alumnos de las carreras de Nutrición, Enfermería, Arte, Ciencias Químicas y Medicina, de la UNC.

El desarrollo metodológico se encuadró en la propuesta de la Investigación-Acción Participativa (IAP) a partir de la simultaneidad del proceso de conocer y de intervenir y la participación de los actores sociales en el estudio y en la acción (Ander-Egg, 2006). Se llevaron a cabo encuentros de modalidad taller de dos horas con las/os escolares de 5 to grado de la institución educativa en días y horas diferentes para cada división (50 niñas/os por grupo), en el momento destinado a las asignaturas especiales. Las dinámicas para cada taller (8 en total) se organizaron de manera secuencial: inicio, un momento de distensión y presentación de integrantes seguido de la elaboración de un diagnóstico participativo sobre el tema a tratar durante la jornada por medio de disparadores lúdicos en actividades grupales; desarrollo, realizado a partir de trabajos grupales, charlas dialogadas y puesta en común de producciones con voces de todos los actores (expresadas ya sea en las producciones o las exposiciones orales); cierre, con una síntesis de lo trabajado y evaluación conjunta al finalizar cada encuentro. Se incluyeron diferentes dispositivos pedagógicos en cada encuentro como utilización de videos, expresión a través del dibujo, "observar con los ojos cerrados para reconstruir los recorridos diarios al colegio", construcción colectiva de conceptos, discusión, debate y plenario integrador, entre otros. Una particularidad de esta experiencia estuvo dada por las actividades lúdicas propuestas, que potenciaron el desarrollo de todos los sentidos (vista, olfato, tacto, audición) transformando el mapeo en proceso vivencial que hizo que su entono tomara vida en los mapas, con colores y olores particulares.

Los Iconoclasistas (2015) definen el mapeo colectivo (MC) como un proceso de creación que subvierte el lugar de enunciación para desafiar los relatos dominantes sobre los territorios, a partir de los saberes y experiencias cotidianas de los participantes. Sobre un soporte gráfico y visual se visibilizan las problemáticas más acuciantes del territorio identificando a los responsables, reflexionando sobre conexiones con otras temáticas y señalizando las consecuencias. El MC como herramienta de observación activa y registro, ofició de eje conductor a lo largo del proyecto. Fue seleccionado por su reconocida capacidad para la construcción de conocimiento local, colectivo y situado (Lafuente y Horrillo, 2017). En la experiencia que presentamos, el MC fue inicialmente utilizado para visibilizar e intercambiar los saberes, las experiencias y situaciones-problema relacionados con el ambiente de vida cotidiana de niñas, niños, docentes y familias.

Atendiendo a los presupuestos de la IAP, los talleres estuvieron sujetos a revisiones y modificaciones que surgieron del consenso entre las/os participantes durante los encuentros. Las actividades fueron documentadas por medio de registros etnográficos (nota de campo) y fotografías (fue efectuado el consentimiento de las familias de las/os niñas y niños), la sistematización de cada encuentro fue un insumo fundamental para la planificación del siguiente, de esta manera las/los niñas/os podían ver reflejadas sus producciones y avanzar en el trabajo propuesto a partir de ello. 


\section{Elaboración colectiva de los conceptos de salud, hogar, ambiente y comunidad}

El primer eje de trabajo abordó los conceptos de salud, hogar, ambiente y comunidad. Cada niña/o dibujó su hogar y su entorno de vida cotidiana. Posteriormente, en grupos de 6 integrantes (5 niñas/os y 1 docente), y a partir de la discusión de las producciones individuales, se elaboró un concepto de salud y se identificó lo que "nos hace bien" y aquello que "no nos hace bien". Se abordaron y asociaron los conceptos de "comunidad" y "ambiente" con lo anteriormente trabajado en cada grupo

Este ejercicio permitió identificar las características del microambiente de vida cotidiana (el espacio, sus habitantes, el hogar y los alrededores de la vivienda). Según las niñas y los niños, la salud es conceptualizada en términos muy amplios, asociada al descanso, la felicidad, la alegría y los lugares para jugar y hacer deportes. En su entorno de vida cotidiana se destaca el canal de riesgo y las quintas. Algunas/os nombran el canal (utilizado para riego de las quintas) cercano a su hogar como "un arroyo bonito", al que asignan atributos positivos, y otros, como un lugar "sucio, lleno de basura y con mal olor", de la mano de las docentes que lo consideran como un lugar peligroso y fuente de contaminación. Para algunas/os niñas/ os, vivir cerca o en las quintas es algo que les puede afectar negativamente por situaciones de violencia que conocen (robo de hortalizas), y por "las avionetas que echan venenos", esto último referido a los campos cercanos de cultivos extensivos. Varios de las/os participantes son hijas/os de horticultores que aplican plaguicidas. Sin embargo, no emergieron reflexiones en torno al tema; en el grupo se percibieron miradas y ausencia de palabras que aborden la situación. En un trabajo previo hemos postulado que en las significaciones que las personas construyen acerca de los plaguicidas, interviene la realidad socioeconómica; aquí, el lugar que las familias de productores/asalariados ocupan en las relaciones mercantiles capitalista de producción (Machado et al., 2012). David Le Breton (2011), plantea que toda percepción del riesgo implica una fuerte connotación afectiva y la retransmisión de un discurso social y cultural. El miedo (en este caso a las intoxicaciones) estaría menos ligado a la objetividad del riesgo que a los imaginarios inducidos. Es posible que las diferentes reacciones de las niñas y los niños acerca de esta problemática hayan estado imbuidas de significaciones compartidas en las familias.

\section{Mapeo de percepciones individuales para integrar conceptos}

El segundo eje apuntó a visibilizar modos y condiciones de vida, a partir de la exploración sensorial de los recorridos habituales de cada niña/o del hogar a la escuela; para posteriormente vincularlo con las concepciones de salud, comunidad y ambiente compartidas en el taller anterior. Se propuso como actividad realizar un "viaje sensorial de la casa a la escuela" para generar los insumos que permitieran elaborar una aproximación a la cartografía. Recurriendo a sus sentidos, se invitó a las/os niñas/os a recrear con la imaginación el mencionado trayecto cotidiano. El/la facilitador/a acompañó la actividad a partir de preguntas guía: ¿en qué momento del día salís de tu casa? ¿Quién te acompaña? ¿Cómo vas, caminando, en bicicleta, etc.? ¿Qué calles, qué espacios cruzas? ¿Sentís olores y ruidos particulares? ¿Qué actividades realizan las personas que ves en el camino? Luego, cada niña/o plasmó sus percepciones en el papel. Una vez concluida esta tarea y pegados los dibujos y/o esquemas en un afiche común al grupo, entre ellas/os diseñaron íconos que pudieran identificar espacios 
comunes para todas/os en el barrio. Así a través del dibujo y el diálogo se logró caracterizar los espacios, prácticas allí desplegadas, y otras percepciones emergentes relacionadas con las personas que habitan los lugares recorridos. Entre los íconos que se diseñaron, encontramos que hojas y pelotas se utilizaron para señalizar plazas y espacios verdes, y una calavera para identificar a los venenos (plaguicidas).

\section{EI MC como herramienta para la construcción del concepto de salud ambiental integral}

Las/os niñas/os construyeron juntos un "mapa vivo de su barrio" a partir de un afiche que contaba con algunas referencias (puntos cardinales, el centro de la ciudad, la calle principal del barrio, el canal, el centro de salud, la escuela). Las preguntas guía fueron: ¿qué puedo identificar de mi ambiente cotidiano que me hace bien y qué puede dañar mi salud? ¿Qué puedo identificar en el ambiente que le hace bien a la comunidad del barrio Guiñazú y qué no le hace bien a mi comunidad? Cada grupo trabajó con un/a facilitador/a que tuvo un rol asignado en la organización, orientación y coordinación. El mapeo elaborado integró los conocimientos construidos, espacios identificados y prácticas percibidas con relación a la salud ambiental.

Las/os participantes se expresaron con claridad respecto de la vinculación entre problemáticas ambientales y las forma en que estas pueden afectar la salud e identificaron aspectos positivos y perjudiciales para su salud. Se asociaron al hecho de estar sanos la posibilidad de contar con espacios verdes y lugares físicos para esparcimiento y recreación y las propuestas en la comunidad para realizar estas actividades: "nos gustaría tener lugares para hacer deportes y cosas divertidas, juntarnos con los amigos" (E1), expresó una compañera.

La tala de árboles en el barrio y el no reemplazo por nuevos se visualizó como un aspecto negativo para el ambiente y la salud en la voz de un niño: "cada día en el barrio tenemos menos árboles y siempre están sacando, a veces para construir algo o porque se ponen viejos y se secan, los sacan y nadie planta uno nuevo" (E2).

Se asoció "la felicidad", o "estar feliz" con la salud, lo cual inicialmente fue planteado por un grupo y recibió gran adhesión por parte del colectivo participante. En los mapas realizados la felicidad se manifestó en los parques, plazas y en la escuela. La investigación en epidemiología social muestra que, dado que somos seres sociales, la comorbilidad de las enfermedades físicas y los trastornos mentales es más alta especialmente en aquellos que manifiestan no sentirse felices (Cacioppo, 2008).

La calidad de la alimentación fue destacada como elemento central para mantener la salud y crecer sanos y se tuvieron en cuenta los vegetales y frutas, el consumo de agua en lugar de bebidas azucaradas y la comida hecha en casa como las mejores opciones. En este sentido, Pedro José Carrillo López et al. (2021) determinaron que existe relación entre la percepción de soledad, felicidad y salud, y la dieta considerada saludable en un estudio con escolares españoles durante el año 2020.

En diferentes lugares del barrio se identificó el fenómeno de la contaminación: "el canal" donde se acumula basura que es arrojada por los vecinos: "es muy feo ver que la gente tira cosas viejas y basura al canal", "encontramos animales muertos y hasta una heladera rota, siempre hay mal olor cerca del canal"; terrenos baldíos donde se acumula basura y se ob- 
servan muchos perros en mal estado de salud: "yo me llevé uno de esos perros que estaba enfermo y vivía en un baldío, lo curé y ahora es mi perro" (E1, E5).

Como se mencionó, las avionetas representan el problema con los plaguicidas, la cercanía de la casa a los campos de cultivos extensivos fue identificada como algo negativo para la salud, aunque no expresaron vivencias que puedan caracterizar lo anterior. En esta instancia nos preguntamos: ¿cómo vivencian esta problemática las/os niñas/os que viven en las quintas? Pero quienes habitan allí evitaron hablar de los problemas de la contaminación con plaguicidas. Conocemos, a partir de la demanda generada por un grupo de mujeres, que este tema genera malestar entre miembros de la comunidad, por lo que es posible que las/os niñas/os eviten hablar de ello como una forma de ocultar o negar el problema al que están expuestos.

Con el propósito de compartir las producciones elaboradas y a partir de ello pensar juntos actividades que respondan a las temáticas emergentes identificadas, familiares y miembros de la comunidad fueron invitados al último encuentro taller. Las/os adultas/os participantes se integraron a los grupos de trabajo para reflexionar y/o discutir juntos las producciones realizadas en los encuentros previos. En la jornada se destacó el protagonismo de las/os jóvenes investigadoras/es y la escucha atenta de las/os adultas/os. Al abordaje de las problemáticas relacionadas con la salud ambiental desde la lente de las/os niñas/os se sumaron observaciones de las/os invitados en el mismo sentido, aportando una mirada temporal al proceso de deterioro del ambiente en detrimento de la salud de la población del barrio. A modo de ejemplo, acerca de la calidad del aire en el barrio, una madre participante expresó: "El aire que respiramos está cada vez más contaminado en el barrio, nací en este acá y antes no era así" (E4, E3, E5, E6, E7, E8, E9, E10), a lo que otro grupo agregó en el momento plenario que el aumento de "humo" (E18, E19, E20, E21, E22, E23) en el barrio es atribuido a la gran cantidad de fábricas y quema de basurales. Se sumó en esta discusión una mirada en torno a los plaguicidas: "también tenemos las fumigaciones cada vez más cerca del barrio" (E5). La necesidad de plantar árboles en la vía pública y su recambio en espacios verdes fue observada por el colectivo como propuesta en lo inmediato para mejorar la calidad del aire que respiran: "si sumáramos árboles en vez de sacarlos, mejoraríamos un poco esta situación" (F1).

\section{Reflexiones para seguir construyendo}

La invitación a ser investigadores barriales en el entorno escolar para contemplar de manera integral la salud ambiental constituyó un elemento idóneo para desarrollar una educación crítica, participativa y transformadora. Esta experiencia invita a reflexionar en torno al aprendizaje situado y las posibles estrategias para abordarlo. Se considera conocimiento situado a aquel que se genera y se recrea en determinada situación (Diaz Barriga Arce, 2006). Existen distintos lugares desde donde mirar la realidad y, por tanto, se hace necesario exponer cuál es nuestra perspectiva. Por eso el conocimiento siempre será parcial y situado. De la articulación de todas las miradas y perspectivas podremos tener un conocimiento más cercano o profundo de la realidad. En este proyecto, ello fue motivado a partir de la invitación a observar y pensar(se) en el ambiente cotidiano a través de la exploración de los sentidos (vista, oído, olfato) utilizando propuestas lúdicas. Respecto de ello nos preguntamos: para realizar 
el abordaje territorial situado acerca de la salud ambiental, ¿es necesario salir al territorio? Y si esto no fuera posible, ¿podemos lograr resultados similares a partir de la implementación de estrategias que recreen el territorio en el aula? La relevancia de la temática para el grupo y el tipo de interacciones que se propicien serían la clave para lograrlo. Algunos autores exponen que experiencias de extensión en torno a temáticas ambientales en territorio logran mayor potencialidad en la promoción del conocimiento y motivación del interés (Sato y Carvalho, 2005; Carvalho, 2006). Y en oposición a ello, Stern et al. (2014) afirman que, si las actividades de extensión se realizan de manera sistemática y adecuada fuera del terreno, pueden alcanzar diferentes niveles de eficiencia promoviendo el conocimiento y el interés en temas ambientales. Se ha criticado el tratamiento de las cuestiones ambientales en los entornos educativos formales por incorporar conceptos y definiciones puramente teóricos y simplificar demasiado el significado del entorno natural, por ejemplo, con relación a la ecología, posicionando al sujeto como un elemento externo al medio (Reigota, 1999; Mendonça, 2007; Loureiro, 2012). Desde estas miradas críticas, se postuló que es muchas veces imposible, desde este posicionamiento que se cuestiona, la formación de valores, mentalidades y actitudes necesarias para el desarrollo de soluciones sentidas por los colectivos humanos en cuanto a la salud ambiental (Sauvé, 2005). En este sentido, Carvalho (Sato; Carvalho, 2005) postuló que la utilización de una buena orientación político-ideológica de la manera de trabajar permite que las/os escolares se posicionen como una parte clave e integral del entorno natural y cambien sus concepciones y perspectivas sobre este contexto (sobre la naturaleza, el rol de las personas, la incidencia de la acción humana en el medio ambiente).

En nuestra experiencia en la escuela de Guiñazú, el despliegue de actividades en el aula que jerarquizó el rol de las/os niñas/os en sus diferentes formas de conocer su territorio, posibilitó traer y recrear el barrio en el aula. Esta perspectiva para la intervención colocó en situación de sujetos activos a las/os niñas/os. Sus diálogos y reflexiones compartidas permitieron negociar y modificar posiciones previas y definir entre todos qué cosas son dignas de transformación; es decir, ocurrieron procesos en los que se construyeron conceptos con significados con relación a las propias posiciones y también a los contenidos a trabajar.

EI MC fue la herramienta que hizo factible establecer la observación y la reflexión de las/os niñas/os con el territorio y ayudó a mitigar, al menos en parte, la fragmentación del conocimiento, al que se le atribuye la comprensión incompleta y falta de interés de las/os escolares cuando, por ejemplo, las problemáticas relacionadas con el ambiente no son visualizadas por ellos mismos en terreno (Souza, 2014). La cartografía social como proceso ha sido validado por su capacidad para reconocer el territorio, identificar y priorizar temáticas y/o situaciones sentidas, y desde allí construir los cimientos para trabajar colectivamente en la búsqueda de respuestas transformadoras (Risler y Ares, 2013). El uso y recreación de herramientas metodológicas adecuadas en el abordaje de las problemáticas vinculadas a la salud ambiental comunitaria dentro del ambiente escolar ha sido fundamental. Brindó insumos para problematizar la relación entre teoría y práctica, entre el conocimiento académico y saber cotidiano, generó rupturas en las ideas de continuidad y dio lugar a la duda, a la repregunta y a la ocasión para nuevos aprendizajes. Es necesario tener en cuenta que el mapa es solo una herramienta y, si su elaboración está inserta dentro de un proceso de organización y articulación colectiva, facilita el diagnóstico y la preparación de proyectos que comprometan la participación y se sostengan en el tiempo. 
El conocimiento que emerge de este proceso participativo planteó problemáticas diferentes de las que motivaron inicialmente este proyecto. Se objetivó que la principal necesidad sentida de las/os niñas/os está relacionada con la posibilidad de contar con espacios para la recreación y propuestas culturales y de participación por parte de las instituciones del barrio, ligado ello a la posibilidad de estar felices (característica que el grupo asoció a la salud). En el equipo extensionista persisten interrogantes respecto de lo que no emergió en el grupo, particularmente en lo vinculado con las problemáticas de los plaguicidas y la pertenencia de varias/os niñas/os a FH. En torno a ello nos preguntamos: ¿la aplicación de plaguicidas y sus posibles consecuencias son visualizados por las/os niñas/os en las $\mathrm{FH}$ ? ¿Es posible que estas no formen parte de sus inquietudes? ¿Estaríamos frente a un tema atravesado por tabúes familiares o sociales?

La jornada de trabajo con adultas/os familiares permitió sumar miradas desde diferentes lugares a la problemática identificada por las/los niñas/os. Se manifestaron pujas territoriales dadas por la dinámica de la instalación de industrias en la interfase rural-urbana del barrio, la dinámica de las quintas hortícolas y la reconversión de estas a la agricultura extensiva, principalmente soja, que genera nuevos conflictos por contaminación con agroquímicos (fumigaciones).

Se rescata que el proceso transitado admitiría generar una agenda de salud para las/os niñas/os en el barrio a partir de la priorización que ellas/os mismas/os realizaron y sumando a otros actores de la comunidad.

Entre nosotras/os, como equipo de docentes investigadores/as extensionistas, la experiencia produjo una fuerte motivación para seguir compartiendo espacios tan fructíferos y gratificantes como este. Luego de años de trabajo en el territorio del cinturón verde desde proyectos de investigación o intervención, centrados en propuestas de prevención compartidas con productoras/es y técnicas/os y diseñados desde metodologías clásicas de la epidemiología/epidemiología comunitaria, esta experiencia dio al equipo la oportunidad de ejercitar, junto a niñas/os y sus docentes, la percepción sensible como forma de aproximación al conocimiento de un espacio territorial. Ello abrió un nuevo horizonte de posibilidades para futuros proyectos y actividades. Así, mientras elaboramos este manuscrito, el contexto de pandemia por COVID-19 impidió realizar actividades a campo. Los aprendizajes recientes en la Escuela Primaria UNESCO apoyaron el diseño de actividades áulicas desarrolladas en virtualidad - vinculadas a la enseñanza del concepto de "territorio" - en la docencia universitaria de grado que forma parte de nuestro quehacer. Persiste, para nosotras/os, el desafío de seguir construyendo y descubriendo modos de aproximación colectiva a territorios y conocimientos.

\section{Referencias bibliográficas}

Ander-Egg, E. (2006). Repensando la Investigación-Acción-Participación. Lumen.

Babbit, S. (2010). El periurbano Sampedrino: un espacio de viveros. En Neiman, G. (Ed.). Globalización y Agricultura periurbana en Argentina (pp. 121-135). Facultad Latinoamericana de Ciencias Sociales (FLACSO). http:// www.flacso.org.ar/wp-content/uploads/2013/11/Globalizacion-y-agricultura-periurbana-en-la-Argentina.pdf

Barchuk, A. H.; Suez, L. S.; Locati, L. (2017). Cobertura y uso de la tierra en el área periurbana de la ciudad de Córdoba, Argentina. Aportes a la planificación territorial. Revista de la Asociación Argentina de Ecología de Paisajes, 7(1), 15-30. 
Bentolila, L. (2018). La planificación estratégica de la extensión universitaria. Los desafíos de construir indicadores para la intervención. +E: Revista De Extensión Universitaria, 7(7), 176-187. https://doi.org/10.14409/ extension.v0i7.7062

Bertolozzi, M. R.; y De la Torre Ugarte Guanilo, M. C. (2012). Salud colectiva: fundamentos conceptuales. Salud Areandina, 1(1), 24-36. Bogotá.

Boulos, M. K.; Resch, B.; Crowley, D.; Breslin, J.; Sohn, G.; Burtner, R.; Pike, W.; Jezierski, E.; Chuang, K. (2011). Crowdsourcing, citizen sensing and sensor web technologies for public and environmental health surveillance and crisis management: trends, OGC, standards and application examples. International Journal of Health Geographics, 10(67).

Breilh, J. (2013). La determinación social de la salud como herramienta de transformación hacia una nueva salud pública (salud colectiva). Revista Facultad Nacional de Salud Pública, 31(1),13-27.

Briggs, D. (1999). Environmental health indicators: framework and methodologies. WHO/SDE/OEH/99.10

Burel, F. y Baudri, J. (2002). Ecología del paisaje, conceptos, métodos y aplicaciones. Ediciones Mundi-Prensa. Butinof, M.; Fernández, R.; Lantieri, M. J.; Stimolo, M. I.; Blanco, M.; Machado, A. L.; Franchini, G.; Gieco, M.; Portilla, M.; Eandi, M.; Sastre, A.; Diaz, M. P. (2014). Pesticides and Agricultural Work Environments in Argentina. En Larramendy, M., Soloneski, S. (Eds). Pesticides -toxic aspects. (pp. 105-134). https://www. researchgate.net/publication/283546513_Pesticides_-_Toxic_Aspects

Cacioppo, J. T. P. (2008). Loneliness: Human Nature and the Need for Social Connection. W. W. Norton y Company.

Carrillo López, P. J.; Guillamón, A. R.; Tárraga López, P. J; García Cantó, E. (2021). Percepción de soledad, felicidad y salud y calidad de la dieta. El papel moderador del estado ponderal. Nutrición hospitalaria, 38(3), 458-463. Carvalho, I.C. de M. (2006). As transformações na esfera pública e a ação ecológica: educação e política em tempos de educação e política em tempos de crise da modernidade. Revista Brasileira de Educação, 11(32), 309.

Casadinho Souza, J. (2005). Intoxicaciones con plaguicidas en niños: impacto en la salud y preparación temprana para el desarrollo de actividades laborales. Centro de Estudios sobre Tecnologías Apropiadas de la Argentina Red de Acción en Plaguicidas y sus Alternativas de América Latina. http://www.aset.org.ar/congresos/7/14005.pdf

Castell, N.; Kobernus, M.; Liu, H.; Schneider, P.; Lahoz, W.; Berre, A.; Noll, J. (2015). Mobile technologies and services for environmental monitoring: The Citi-Sense-MOB approach. Urban Climate, 14(3), 370-382.

Castellanos, P. L. (1990). Los Modelos Explicativos Del Proceso Salud-Enfermedad: Los Determinantes Sociales. Bol Epidemiológico OPS, 10(4).

Diaz Barriga Arceo, F. (2006). Enseñanza Situada: Vínculo entre la escuela y la vida. McGraw Hill Interamericana. Díaz Terreno, F. (2011). Los territorios periurbanos de Córdoba entre lo genérico y lo específico. Revista Iberoamericana de Urbanismo, 5, 65-84.

Eandi, M. A.; Soria, V.; Dezzotti, L.; Butinof, M. (2018). Experiencia participativa orientada a la prevención de la exposición a plaguicidas en la producción hortícola del Cinturón Verde de la Ciudad de Córdoba. +E: Revista de Extensión Universitaria, 8(9), 183-194. https://doi.org/10.14409/extension.v8i9.Jul-Dic.7855

Eandi M. A.; Dezzotti, L.; Butinof, M. (2021). Exposición a plaguicidas y cuidados de la salud en la horticultura periurbana: el caso del Cinturón Verde de la Ciudad de Córdoba, Argentina. Ciênc. saúde coletiva, 26(4). https://doi.org/10.1590/1413-81232021264.27922018

Eandi, M. A. (2020). Condiciones de Salud-Enfermedad y Estrategias de cuidado de la salud de las familias de pequeños productores y trabajadores hortícolas del cinturón verde de la ciudad capital de Córdoba, expuestos a plaguicidas. [Tesis doctoral de Ciencias de la Salud]. Secretaría de Graduados en Ciencias de la Salud. Facultad de Ciencias Médicas. Universidad Nacional de Córdoba. 
Gálvez, M.; Forman, J.; Landrigan, P. J. (2010). Niños. En Frumkin, H. (Ed.). Salud Ambiental. De lo global a lo local (pp. 887-928). OPS.

Giarracca, N. y Teubal, M. (2010) Disputas por los territorios y recursos naturales: el modelo Extractivo. ALASRU Nueva Época, (5).

Giobellina, B. L. (2018). La alimentación de las ciudades. Transformaciones territoriales y cambio climático en el cinturón verde de Córdoba. Ediciones INTA. https://repositorio.inta.gob.ar/xmlui/handle/20.500.12123/4223 Gómez López, L.; Rabanaque Hernández, M. J. (2000). Concepto de salud. En Colomer Revuelta C.; ÁlvarezDardet, C. Promoción de la salud y cambio social. Masson.

Iconoclasistas (2015). Manual de mapeo colectivo: Recursos cartográficos críticos para procesos territoriales de creación colaborativa. Tinta Limón.

Kleffel, D. (1996). Environmental paradigms: Moving toward an ecocentric perspective. Advances in Nursing Science, (18),1-10. http://dx.doi.org/10.1097/00012272-199606000-00004

Lafuente, A. y Horrillo, P. (2017). Cómo hacer un mapeo colectivo (La aventura de aprender). Continta me tienes.

Landrigan, P. J.; Kimmel, C. A., Correa, A., Eskenazi, B. (2004). Children's health and the environment: public health issues and challenges for risk assessment. Environmentak Health Perspectives, 112(2), 257-65.

Le Breton, D. (2011). Conductas de riesgo. De los juegos de la muerte a los juegos de vivir. Buenos Aires: Topía. Loureiro, C. F. B. (2012). Trajetória e fundamentos da educação ambiental. 4ta. ed. Cortez.

Lupton, D. (2015). Health promotion in the digital era: a critical commentary. Health Promotion International, $30(1), 174-183$.

Machado, A. L.; Butinof, M., Sastre, A. (2012). Estilos de vida y percepción de riesgo en prácticas con plaguicidas. Poiésis, 12(23). http://www.funlam.edu.co/revistas/index.php/poiesis/article/viewFile/343/321

Machado, A. L.; Butinof, M.; Eandi, M.; Portilla, A.; Fernández, R. A.; Soria, V.; Franchini, G. (2017). Vulnerabilidad y riesgo por plaguicidas en horticultura del cinturón verde en Córdoba, Revista Facultad Nacional de Salud Pública, 35(1).

Mendonça, R. (2007). Educação Ambiental vivencial. En Ferraro Júnior, L. A. (Org.). Encontros e Caminhos: Formação de Educadoras (es) Ambientais e Coletivos Educadores. MMA, Diretoria de Educação Ambiental, (2) (pp. 116-129).

National Research Council (1993). Pesticides in the Diets of Infants and Children. http://www.nap.edu/catalog/2126.html.

Nicholson, R. (2001). Environmental health practice: For today and for the future. Environmental Health, (1), 73-84.

Organización Panamericana de la Salud (OPS) (2005). Metodologías de evaluación de riesgo y de impacto a la salud en México. (2/09/2008). http://www.mex.opsoms.org/contenido/metodologia.htm

Ordoñez, G. A. (2000). Salud ambiental: conceptos y actividades. Revista Panamericana de Salud Pública, 7(3), 137-47.

Organización Mundial de la Salud (OMS) (1994). Salud, medio ambiente y desarrollo; enfoques para la preparación de estrategias a nivel de países para el bienestar humano. Ginebra.

Plowright, R. K.; Sokolow, S. H.; Gorman, M. E.; Daszak, P.; Foley, J. E. (2008). Causal inference in disease ecology: Investigating ecological drivers of disease emergence. Frontiers Ecology Environment, 6, 420-9. http://dx.doi.org/10.1890/070086

Reigota, M. (1999). Ecologia, elites e intelligentsia na América Latina: um estudo de suas representações sociais. Annablume. 
Risler, J. y Ares, P. (2013). Manual de mapeo colectivo: recursos cartográficos críticos para procesos territoriales de creación colaborativa. Tinta Limón.

Sato, M.; Carvalho, I. C. de M (Orgs.) (2005). Educação ambiental: pesquisa e desafios. Artmed.

Sauvé, L. (2005). Uma cartografia das corrientes em educaçao ambiental. En Sato, M.; Carvalho, I. C. de M. (Orgs.). Educação ambiental: pesquisa e desafios (pp. 17-46). Artmed.

Senabre, E.; Ferran-Ferrer, N.; Perelló J. (2018). Diseño participativo de experimentos de ciencia ciudadana. Comunicar, XXVI(54), 29-38.

Souza, M. C. C. (2014). Educação Ambiental e as trilhas: contextos para a sensibilização ambiental. Revista Brasileira de Educação Ambiental (REVBEA), 9(2), 239-253.

Stern, M. J.; Powell, R. B.; Hill, D. (2014). Environmental education program evaluation in the new millennium: what do we measure and what have we learned? Environmental Education Research, 20(5), 581-611.

Wild, C. P.; y Kleinjans, J. (2003). Children and Increased Susceptibility to Environmental Carcinogens. Cancer Epidemiology, Biomarkers \& Prevention, 12, 1389-1394. 